Meta

Journal des traducteurs

Translators' Journal

\title{
Index of Words and Topics in Volume 17
}

Volume 17, numéro 4, décembre 1972

URI : https://id.erudit.org/iderudit/003185ar

DOI : https://doi.org/10.7202/003185ar

Aller au sommaire du numéro

\section{Éditeur(s)}

Les Presses de l'Université de Montréal

ISSN

0026-0452 (imprimé)

1492-1421 (numérique)

Découvrir la revue

Citer ce document

(1972). Index of Words and Topics in Volume 17. Meta, 17(4), 268-271.

https://doi.org/10.7202/003185ar d'utilisation que vous pouvez consulter en ligne.

https://apropos.erudit.org/fr/usagers/politique-dutilisation/ 


\section{INDEX OF WORDS AND TOPICS IN VOLUME 17}

Abridgement, 3, p. 161.

Accentual-syllabic verse system, 2, p. 104.

Accentual verse system, 2, p. 104.

Access to information, 4, p. 204.

Access word, 4, p. 205.

Adjectives used for describing translations, 2, p. 95 .

Advance planning in language services, 3,

$$
\text { p. } 145 .
$$

Advertising, 1, p. 7 , p. 78

Afro-american slang, 3, p. 193.

Agency secretary, 3, p. 168.

Agency shop, 3, p. 166.

Agent of record, 3, p. 168.

Agreement, 2, p. 126.

Agricultural trade, 2, p. 129.

A.I.D.A., 1, p. 8.

American Comparative Literature Association, 2 , p. 97 .

American usage in automotive terminology, 2, p. 118.

Ammeter, 2, p. 118 .

Analog quantity, 3, p. 189.

Annual premium value, 3, p. 168 .

Anti-union shop, 3, p. 165 .

Approach to criticality, 3, p. 189.

Arc welding, 3, p. 172 .

Assessments, 4, p. 226.

Assets, 4, p. 241

Author code, 4, p. 213.

Autogenous welding, 3, p. 174.

Automobile, 2, p. 118.

Automotive terminology, 2, p. 118.

Auxiliary files, 4, p. 205

Axle journals, 3, p. 188 .

Axle spindle, 3 , p. 188 .

Back-up files, 4 , p. 219.

Balance (to) an account, 4, p. 241.

Balance of an account, 4, p. 241.

Banana belt, 3, p. 185 .

Banking account, 4 , p. 241.

Bank-note, 2, p. 89 .

Bargain, 2, p. 111.

Bargainer, 2, p. 111.

Bargaining, 2, p. 111

Bath towel, 3, p. 191.

Bird's eye view, 4, p. 208.

Book review, 2 , p. 94 .

Book review commentary, 2, p. 96

Bow, 2, p. 115.

Box, 2, p. 114 .

Brainstorming, 4, p. 230.

Branch secretary, 3, p. 169.

Braze welding, 3, p. 176 .

Brazing, 3, p. 171 .

British usage in automotive terminology, 2, p. 118.

Purden of translation research, 4, p. 206.

Business and professional correspondance, 2 , p. 130.

Business games, 4, p. 230.
Canadian cuisine, 3, p. 191.

Canadian English, 3, p. 181, p. 195.

Canadian usage in automotive terminology, 2 , p. 118.

Car-ferry, 2, p. 88.

Car radio, 2 , p. 118 .

Case study, 4, p. 230

Casting, 2, p. 114

Ceramic terms, 4, p. 249.

Charge indicator, 2 , p. 118.

Chargex, 1, p. 65 .

Checkoff, 4, p. 225.

Chronological English Dictionary, 3, p. 193.

Clean as a whistle, 1, p. 23.

Cloud chamber, 3 , p. 188.

Club, 3, p. 191.

Coaching, 4, p. 228.

Codes used in data unit, 4 , p. 212.

Column, 2, p. 118.

Communication process, 2, p. 102.

Compact car, 3, p. 191.

Comparison of translations, 2, p. 96.

Comparison of translation styles, 3 , p. 160

Compulsory checkoff, 4, p. 225.

Computer operation, 4, p. 205.

Condenser, 3, p. 187.

Conference, 4, p. 230

Conference leader, 4, p. 230.

Conference training, 4, p. 229.

Consecutive interpreting, 3, p. 148.

Contact, 2, p. 117 , p. 119.

Container, 2 , p. 88, p. 113 .

Contextual uses, 3 , p. 182.

Continuation training, 4, p. 227.

Continuing education, 4, p. 227.

Conversational mode, 4, p. 205.

Correction data unit, 4, p. 214.

Craft training, 4, p. 227

Crane, 2, p. 115.

Crate, 2, p. 113.

Cream rinse, 1 , p. 57 .

Creative thinking, 4, p. 230.

"Crib ", 2, p. 95.

Criteria for selecting interpreters, 3, p. 149.

Criteria of book reviewing, 2, p. 94 .

Criterion of relevance, 2 , p. 99.

Criticality, 3, p. 189.

Cross-pollination, 3, p. 191.

Cross-temporal factor, 2 , p. 102.

Crushing injury, 4, p. 239.

Data unit content, 4 , p. 209.

Daily mileage indicator, 2 , p. 118 .

Dead man, 3, p. 191.

Dead storage, 3, p. 191 .

Debenture, 4 , p. 241 .

Decentralization of language services, 3, p. 144

Declaration language, 4 , p. 208 .

De-energize, 3, p. 188 .

Deep-pile, 1, p. 24.

Defalcate, 3 , p. 185 
Definition, 3, p. 182.

Defogger, 2, p. 118 , p. 119.

Defrost, 2, p. 118, p. 119

Defroster, 2, p. 118, p. 119.

Delays in disseminating terminological information, 4, p. 207.

Demist, 2, p. 118, p. 119

Deodorant, 1, p. 27.

Derivatives, 3 , p. 184

Design, 2, p. 90 .

Development, 4, p. 227.

Diachronic factor, 2 , p. 102

Diatonic scale, 3 , p. 185 .

Direct access, 4, p. 204

Directional signal, 2 , p. 118.

Directional signal switch, 2, p. 118

Director, 3, p. 167.

Director of marketing, 3, p. 168.

Discipline, 3, p. 191.

Discounter, 1, p. 74.

Disparities in value of research, 4, p. 206

Dispersal of research effort, 4, p. 206.

Displacement, 3, p. 162.

Dog, 3, p. 183.

Door, 2, p. 114

Drinking water, 3, p. 191.

Dues, 4, p. 225 .

Dues collection, 4, p. 225 .

Early or traditional modern, 2, p. 105.

Editor, 3, p. 146.

Educating the user, 3, p. 144.

Effective range, 3, p. 190.

Eh, 3, p. 185.

Electric phonograph, 3, p. 171.

Embellishments of a book review, 2, p. 100.

English sentence structures, 3, p. 195.

Entrance examination, 3, p. 150.

Entry, 4, p. 205, p. 208.

Error of emphasis in translation review, 2, p. 97.

Escape clause, 3, p. 167.

Esculent, 3, p. 185 .

Evidence of usage, 4, p. 204.

Exclusive bargaining agent, 3, p. 166.

Executive officers, 3 , p. 167

Experimental modern, 2, p. 105.

Experiments in language translation, 3, p. 178.

Extrapolated range, 3, p. 190.

Eye, 2, p. 114.

Faithfull translation, 3, p. 160.

Familiarization program, 3, p. 150.

Field directory, 4, p. 205.

Field indication, 4, p. 204.

Field man, 3, p. 169

Field organization, 3, p. 169

Fields classification, 4, p. 209

First-line supervision, 4, p. 227

Fitting, 2, p. 114.

Florist shop, 3, p. 191.

Flying sqadron training, 4, p. 228.

Fog, 3, p. 183.

Footer, 2, p. 115.

Foremen, 4, p. 228.

Formal model of translation, 2, p. 102.

Frame, 2, p. 113

Free translation, 3, p. 160

Frequency response, 4, p. 208

Fresh, 3, p. 184.
Functive verb, 3, p. 163.

Fusion welding, 3, p. 174.

Gear change lever, 2, p. 118.

General manager, 3, p. 168.

Gramophone, 3, p. 170 .

Graphic disparity, 4, p. 205

Group discussion, 4, p. 230 .

Group dynamics, 4, p. 229.

Group training, 4, p. 230.

Guided experience, 4, p. 228.

Guiding principles in terminological works, 4, p. 207.

Half-life, 3, p. 188.

Hard soldering, 3 , p. 171 , p. 176

Headlamp, 2, p. 118

Headlight, 2, p. 118.

Headlight switch, 2, p. 118.

Head office underwriter, 3, p. 169.

Head of language services, 3, p. 143

High life, 2, p. 89.

Historicizing translation, 2, p. 105.

Hold-up, 3, p. 185.

Homograph, 3, p. 183.

Homophone, 3 , p. 183

Huh, 3, p. 185 .

Hygiene, 3, p. 186.

Hygienic, 3, p. 186

Ignition switch, 2, p. 117.

Inaccessibility of information, 4, p. 206.

In-house translation, 3, p. 146.

Tnitiation fees, 4, p. 226

Inquiry language, 4, p. 218 .

Instructor, 4, p. 228.

International phonetic alphabet, 3, p. 182.

International treaties, 2 , p. 130 .

Interpreter training, 3, p. 150.

Interpreting, 3, p. 147.

Interpreting services, 3 , p. 147.

Interpreting test, 3, p. 150 .

Inter-system incompatibilities, 2, p. 103.

Intra-language gap, 3 , p. 164 .

Issuing, 3, p. 168.

Jay-walker, 1, p. 66.

J.I.T., 4 , p. 228 .

Job instruction training, 4, p. 228

Job methods training, 4 , p. 228 .

Job relations training, 4, p. 229.

Job safety training, 4 , p. 229.

Journalistic function of literary criticism, 2, p. 95 .

J.S.T., 4, p. 229

Judgments of a translation, 2, p. 95.

Keywords, 4, p. 204.

Kickshaw, 3, p. 184

Kissing spine, 4, p. 240.

Kissing ulcer, 4, p. 239.

Laboratory system, 3 , p. 188.

Laboratory training, 4, p. 229.

Lamp, 2, p. 119.

Language notation, 4, p. 205.

Laud, 3, p. 183.

Leader, 4, p. 228.

Leadership training, 4, p. 229.

Leasing, 1, p. 75 .

Lecture, 4 , p. 230.

Lexicography, 4, p. 204.

Light, 2 , p. 119.

Light brazing, 3, p. 172. 
Limitation of space in book reviewing, 2, p. 95. Line integral, 3, p. 189.

Literary criticism, 2, p. 94.

Living-room, 2, p. 92.

Lock bar, 2, p. 114

Log, 3, p. 183 .

Logic design of data unit, 4, p. 213

Look-up mode, 4, p. 204.

Look-up statistics, 4, p. 218.

Lower management, 4, p. 227.

Luxurious, 1, p. 34.

Maintenance of membership, 3, p. 166

Management, 2, p. $92 ; 4$, p. 227.

Management decision simulation, 4, p. 230.

Management games, 4, p. 230.

Manager, 3, p. 168 .

Manic, 3, p. 186.

Marketing, 1 , p. 7 , p. $45 ; 2$, p. 92 .

Mass generation of input data, 4, p. 206.

Mass input generation, 4, p. 215.

Meaningfulness of context, 4, p. 208.

Meanings, 3, p. 182.

Mechanical recorder, 3, p. 170.

Meeting, 4, p. 230.

Member in good standing, 4, p. 226

Metapoem, 2, p. 107.

Metapoetic process, 2, p. 103.

Meteorology, 2, p. 129

Methectics, 4, p. 229.

Middle management, 3 , p. $167 ; 4$, p. 228 .

Mileage indicator, $2, \mathrm{p} .118$.

Mileameter, 2, p. 118.

Mining, 2, p. 129

Mirror nuclei, 3, p. 189.

Modernizing translation, 2, p. 105

Modern Language Association of America, 2, p. 97.

Morphology, 3, p. 185.

National Council of Teachers of English, 2, p. 97.

Nationhood, 3, p. 191

Native informant, 3, p. 147.

Native speaker as translator, 3, p. 146.

Native town, 3, p. 191.

Nitchie, 3 , p. 185 .

Notation of date, 4, p. 209.

Objectives of the Terminology Bank, 4, p. 215.

Octane number, 3, p. 188.

Odometer, 2, p. 119.

Officer, 3, p. 168.

Officers, 3 , p. 167

Off-the-job training, 4, p. 227.

Olympic games, 3 , p. 143.

Olympic Languages, 3 , p. 148.

On-line operation, 4 , p. 218

On-line updating, 4, p. 218.

On-the-job training, 4, p. 227.

Operation of the Terminology Bank, 4, p. 206.

Oral test, 3, p. 150.

Organizational structure, 3 , p. 145.

Original, 3, p. 191.

Outside contracting, 3 , p. 146

Partition, 3, p. 189.

Patched-up translation, 2, p. 120

Performance of the translator, 2, p. 94.

Permutation of serial components, 3, p. 162.

Phonograph, 3, p. 170.

Pick-up, 3, p. 170, p. 171.
Pick-up stylus, 3, p. 171.

Pipe-line, 2, p. 87.

Placed under the risk, 3, p. 168.

Placing, 3, p. 168.

Planner, 3, p. 146.

Plant, 4, p. 204

Poetic system, 2, p. 103.

"Pony ", 2, p. 95.

Power train, 1, p. 16

Preferential shop, 3, p. 166

Pressure welding, 3, p. 176.

Probation period, 3, p. 167

Production process in language services, 3, p. 146.

Production schedules, 3 , p. 145.

Professional background of a head of language services, 3 , p. 143

Professional language services, 3, p. 144

Programmed instruction, 4, p. 230 .

Programmed learning, 4, p. 230.

Pronunciation key, 3, p. 182.

Pronunciation system, 3, p. 182.

Prospect, 1, p. 8.

Pseudo data unit, 4, p. 214.

Put a tiger in your tank, 1 , p. 35 .

Quality control in language services, 3, p. 144

Quantity of electricity, 3, p. 188.

Quick-freezing, 3, p. 188.

Radiator, 2, p. 118 .

Radioset, 2, p. 118 .

Rand Formula, 3, p. 166.

Rationale, 1 , p. 10 , p. $24 ; 4$, p. 204

Ratio of translator per editor, 3, p. 146

Reading, 4, p. 230.

Re-capture, 2, p. 126.

Record player, 3, p. 171

Re-creative translation, 2, p. 105.

Recruiting interpreters, 3 , p. 149.

Redound, 3, p. 185 .

Reference frequency, 4, p. 208

Reference to source, 4, p. 208.

Regional restriction, 3, p. 184 .

Reliability of information, 4, p. 208.

Resistance to modernizing, 2, p. 109.

Retentive translation, 2, p. 105 .

Retraining, 4, p. 229.

Reversing device, 2, p. 118.

Reviewing translations, 2, p. 94.

Revisor, 3 , p. 146

Ring, 2, p. 114.

Role playing, 4 , p. 230

Rondel, 2, p. 104.

Rot, 3, p. 183.

Run-on entries, 3, p. 185.

School dictionary, 3 , p. 181 .

Scrapper, 3, p. 191.

Scratch, 1, p. 26.

Secretary, 3, p. 168

Seminar, 4, p. 230.

Sensitivity training, 4, p. 229.

Sequential operation, 4, p. 218

Sequential processing, 4, p. 205.

Shift lever, 2 , p. 118

Shop, 3, p. 165.

Simultaneous interpretation, 3, p, 148.

Slob ice, 3, p. 185

Socio-cultural system, 2, p. 103.

Soft soldering, 3, p. 171 , p. 176. 
Soldering, 3, p. 171, p. 176.

Solutions to terminological problems, 4, p. 207. Soudobrasage, 3 , p. 176

Spacer, 2, p. 115

Spanish-English lexicology, 3, p. 193.

Standard archaic usage, 3 , p. 105

Standard translation difficulties, 2, p. 96.

Stative verb, 3, p. 163.

Steering column, 2, p. 118 .

Stick-up, 3, p. 185.

Storage, 4, p. 206.

Superintendant of agencies, 3 , p. 168 .

Support, 2, p. 115 .

Switch, 2, p. 122.

Switch on, 2 , p. 117

Syllabic verse system, 2, p. 104.

Systematic grammar, 3, p. 195

System description, 4, p. 203

System operation, 4 , p. 214

Tales of Kipling, 3, p. 186.

Tank, 2, p. 87.

Teaching machine, 4, p. 231

Teamwork, 3, p. 147

Temporal dialect, 2 , p. 105

Temporal restrictions, 3, p. 184

Terminological directory, 4, p. 204

Terminological parameters, 4, p. 209.

Terminology bank, 4, p. 203.

Terminology data unit, 4, p. 209.

Termium file, 4, p. 204.

Thermionic, 3, p. 188

T-group training, 4, p. 229

Tiger, 1, p. 35.

Timepiece, 1, p. 16.

Time sharing, 4, p. 204

Top management, 3, p. 167.

Trade training, 4, p. 227.

Traditional modern, 2, p. 105.

Trainer, 4, p. 228.

Training, 4, p. 227 .

Training administrator, 4 , p. 228 .

Training department, 4, p. 228.

Training development program, 4, p. 228.

Training director, 4, p. 228.

Training division, 4 , p. 228.

Training group, 4 , p. 229

Training needs, 4 , p. 228.

Training policy, 4, p. 228.

Training specialist, 4 , p. 228

Training staff, 4, p. 228.
Training techniques, 4, p. 228.

Training within industry, 4, p. 228.

Translating staff, 3 , p. 146

Translation as source of terminological data, 4, p. 208.

Translation review, 2 , p. 94.

Translation review in North America, 2, p. 97.

Treatment, 4, p. 204.

Trip mileage indicator, 2 , p. 118 .

Tunnel, 2, p. 114

Turn signal, 2 , p. 118

Turn-table, 3, p. 171.

Tweeter, 1 , p. 77

Underwriting secretary, 3, p. 168.

Unglided vowels, 3 , p. 182 .

Union dues, 4, p. 226.

Union member, 3, p. 166

Union membership, 3, p. 165 .

Union security, 3, p. 165 .

Union shop, 3, p. 167.

Unsatisfactoriness of translations, 2, p. 98.

Updating and special input generation, 4, p. 216.

Usage labels, 3, p. 185 .

Usage notes, 3, p. 181 .

Validity of terminological information, 4 p. 207.

Velocity operated type microphone, 4, p. 208

Versatilily training, 4 , p. 228.

Verse translation, 2, p. 102

Vocational rehabilitation, 4, p. 229.

Vocational training, 4, p. 227

Voluntary checkoff, 4, p. 225.

Wall, 2, p. 114 .

Water waste treatment plant, 4 , p. 204

Week-end, 2, p. 92.

Weighting index, 4, p. 209.

Welding, 3 , p. 172 .

Wheel, 2, p. 115 .

Whistler, 3, p. 185.

Windscreen, 2 , p. 118

Windshield, 2, p. 118.

Woofer, 1, p. 77

Word bank, 4, p. 203.

Written business, 3, p. 167.

Wrongheadedness in translation review, 2, p. 97.

Wrought, 3, p. 183

Yearbook of Comparative and General Literature, 2, p. 97.

Zipper, 3, p. 191 